S. Nagaoka

Nagoya Math. J.

Vol. 71 (1978), 43-60

\title{
P-ADIC PROPERTIES OF SIEGEL MODULAR FORMS OF DEGREE 2
}

\author{
SHŌYŪ NAGAOKA
}

\section{Introduction}

H. P. F. Swinnerton-Dyer determined the structure of the algebra of modular forms $\bmod p$ for all prime numbers $p$ in elliptic modular case (cf. [10]). Using his result, J.-P. Serre investigated the properties of $p$-adic modular forms and succeeded to construct the $p$-adic zeta functions for any totally real number fields (cf. [8]).

In this paper, we shall try to generalize the result of SwinnertonDyer to the Siegel modular case.

In Part I, we shall study the property of Eisenstein series of degree 2.

Our result is stated as follows:

THEOREM. Let $\Psi_{k}$ be the Eisenstein series of degree 2 and of weight k. Let $Z_{m}$ denote a numerator of the $m$-th Bernoulli number $B_{m}$. We assume that the prime number $p \neq 2,3$ satisfies $Z_{p-3} \neq 0(\bmod p)$. Then

$$
\Psi_{k} \equiv 1\left(\bmod p^{m}\right) \Leftrightarrow k \equiv 0\left(\bmod p^{m-1}(p-1)\right) \text {. }
$$

(Furthermore we have gotten the similar result in the case of arbitrary degree $n$, which will be stated in Part I.)

In Part II, we shall generalize the notion of the algebra of modular forms $\bmod p$ to the case of Siegel modular forms of degree 2 , and determine its structure.

We shall begin with the definition of Siegel modular forms $\bmod p$. It is well known that the Siegel modular form $f(Z)$ of degree 2 has a Fourier expansion of the form

$$
f(Z)=\sum_{T \geqq 0} a(T) \exp \{2 \pi i \operatorname{tr}(T Z)\}
$$

Received June 10, 1977. 
where $T$ runs over all half integral positive semi-definite symmetric matrices of degree 2. Denote by $\bigcap_{p}$ the local ring of $\boldsymbol{Q}$ at $p$, i.e. the ring of all rational numbers with denominators prime to $p$. Let $\boldsymbol{I}_{k, p}$ be the $\mathfrak{D}_{p}$-module of Siegel modular forms of degree 2 with even weight $k$ whose Fourier expansions have all their coefficients in $\mathfrak{S}_{p}$, and let $\tilde{I}_{k, p}$ be the space of all formal power series

$$
\tilde{f}=\sum \widetilde{a(T)} \exp \{2 \pi i \operatorname{tr}(T Z)\}
$$

where $f(Z)=\sum a(T) \exp \{2 \pi i \operatorname{tr}(T Z)\}$ runs over all the elements of $\boldsymbol{I}_{k, p}$ and the tilde denotes the reduction $\bmod p$. Then we can define the $\boldsymbol{F}_{p^{-}}$ algebra $\tilde{M}_{2}$ of modular forms $\bmod p$ of degree 2 by $\tilde{M}_{2}=\sum_{k: \text { even }} \tilde{I}_{k, p}$.

Our main result can be stated as follows :

Let $\chi_{10}$ and $\chi_{12}$ are Siegel modular forms of degree 2 and of weight 10 and 12 respectively, which will be defined in Part I.

MAIN THEOREM. Let $\Psi_{k}$ be the same as in the above theorem. Let $p \neq 2,3$ be a prime number satisfying $\Psi_{p-1} \equiv 1(\bmod p)$. Then

$$
\tilde{M}_{2} \cong \boldsymbol{F}_{p}[U, V, W, X] /(\tilde{B}-1) .
$$

Here $B$ is the polynomial with coefficients in $\mathfrak{D}_{p}$ satisfying $\Psi_{p-1}=B\left(\Psi_{4}\right.$, $\Psi_{6}, \chi_{10}, \chi_{12}$ ) and $\tilde{B}$ is the reduction $\bmod p$ of $B$. The isomorphism is induced by corresponding $U, V, W$ and $X$ to $\tilde{\Psi}_{4}, \tilde{\Psi}_{6}, \tilde{\chi}_{10}$ and $\tilde{\chi}_{12}$, respectively.

The author wishes to express his hearty thanks to Prof. Y. Morita and Prof. T. Oda for their valuable advices.

\section{Notations}

We denote by $Z, Q, C$ the ring of rational integers, the field of rational numbers, and the field of complex numbers, respectively.

For any prime number $p$, let $\boldsymbol{Q}_{p}, Z_{p}$ and $\boldsymbol{F}_{p}$ be the field of $p$-adic numbers, the ring of $p$-adic integers, and the finite field with $p$ elements.

We denote by $M_{n}(C)$ the ring of all matrices of size $n$ with entries in $C$. For any element $A$ of $M_{n}(C)$, we denote the trace of $A$ and the determinant of $A$ by $\operatorname{tr}(A)$ and $\operatorname{det}(A)$, respectively.

For a complex symmetric matrix $Z$, we write $Z>0$ (resp. $Z \geqq 0$ ) if $Z$ is positive definite (resp. positive semi-definite).

$\boldsymbol{H}_{n}$ denotes the Siegel upper half plane of degree $n$, namely the space of all complex symmetric matrices $Z=X+i Y$ of degree $n$ with 
imaginary parts $Y>0$.

We denote by $\Gamma_{n}(=\operatorname{Sp}(n, Z))$ the homogeneous Siegel modular group of degree $n$.

\section{Part I}

\section{§1. Siegel modular forms}

In this section, we shall recall the fundamental properties of Siegel modular forms.

First, we define the Siegel modular form of degree $n . \quad \Gamma_{n}=\operatorname{Sp}(n, Z)$ operates on $\boldsymbol{H}_{n}$ by

$$
H_{n} \ni Z \mapsto \sigma(Z)=(A Z+B)(C Z+D)^{-1}
$$

for $\sigma=\left(\begin{array}{ll}A & B \\ C & D\end{array}\right) \in \Gamma_{n}$ with $A, B, C$ and $D \in M_{n}(Z)$.

A holomorphic function $f(Z)$ on $\boldsymbol{H}_{n}$ is called a Siegel modular form of weight $k$ if it satisfies the following conditions :

(1) For every element $\sigma$ of $\Gamma_{n}, f(Z)$ satisfies

$$
f(\sigma(Z))=\operatorname{det}(C Z+D)^{k} f(Z)
$$

(2) $f(Z)$ is bounded in any domain $\left\{Z \mid Y \geqq Y_{0}>0\right\}$ in the case $n=1$. It is well known that $f(Z)$ has the Fourier expansion of the form

$$
f(Z)=\sum_{T \geqq 0} a(T) \exp \{2 \pi i \operatorname{tr}(T Z)\}
$$

where the sum extends over all half integral positive semi-definite symmetric matrices.

The Eisenstein series of degree $n$ and of weight $k$ is defined as follows ;

$$
\Psi_{k}(Z)=\sum \operatorname{det}(C Z+D)^{-k}, \quad Z \in \boldsymbol{H}_{n}
$$

The sum extends over all inequivalent bottom rows of elements of $\Gamma_{n}$ with respect to left multiplications by unimodular integer matrices of degree $n$.

In [9], Siegel gave the formula for the coefficients of Fourier expansion of Eisenstein series.

For a modular form $f(Z)$ of degree $n$, we put 


$$
\Phi(f)\left(Z_{1}\right)=\lim _{\lambda \rightarrow \infty} f\left(\begin{array}{cc}
Z_{1} & 0 \\
0 & i \lambda
\end{array}\right) \quad Z_{1} \in \boldsymbol{H}_{n-1}
$$

Then $\Phi$ maps modular forms of degree $n$ to modular forms of degree $n-1$ of the same weight and it is called the Siegel's operator. If $f(Z)$ is a modular form of degree $n$ and $a(T)$ are its Fourier coefficients, then the Fourier coefficients of $\Phi(f)\left(Z_{1}\right)$ are given by $a\left(T_{1}\right)=a\left(\begin{array}{cc}T_{1} & 0 \\ 0 & 0\end{array}\right)$. In particular, Eisenstein series are mapped by $\Phi$ to Eisenstein series. The Siegel's operator $\Phi$ gives rise to a homomorphism of the graded rings of modular forms.

A modular form is called a cusp form if it is in the kernel of $\Phi$. Here, for the Eisenstein series $\Psi_{k}$ of degree 2, we shall put

$$
\begin{aligned}
& \chi_{10}=2^{2} \cdot 3^{-5} \cdot 5^{-2} \cdot 7^{-1} \cdot 53^{-1} \cdot 43867\left(\Psi_{4} \Psi_{6}-\Psi_{10}\right), \\
& \chi_{12}=2^{-13} \cdot 3^{-7} \cdot 5^{-3} \cdot 7^{-2} \cdot 337^{-1} \cdot 131 \cdot 593\left(3^{2} \cdot 7^{2} \Psi_{4}^{3}+2 \cdot 5^{3} \Psi_{6}^{2}-691 \Psi_{12}\right) .
\end{aligned}
$$

Then these are cusp forms of degree 2 and of respective weight 10 and 12.

For two Siegel modular forms with rational Fourier coefficients $f(Z)=\sum a_{f}(T) \exp \{2 \pi i \operatorname{tr}(T Z)\}$ and $f^{\prime}(Z)=\sum a_{f^{\prime}}(T) \exp \{2 \pi i \operatorname{tr}(T Z)\}$ and for any rational integer $a$, we write

$$
f \equiv f^{\prime}(\bmod a)
$$

if $a_{f}(T) \equiv a_{f}(T)(\bmod a)$ for all $T$.

\section{§2. Congruence properties of Eisenstein series}

Let $E_{k}$ be the normalized Eisenstein series of degree 1 and of weight $k$. It is known that the Eisenstein series $E_{k}$ satisfies following properties (cf. [8]).

$$
\begin{aligned}
& E_{k} \equiv 1\left(\bmod p^{m}\right) \Leftrightarrow k \equiv 0\left(\bmod p^{m-1}(p-1)\right) \quad p \neq 2, \\
& E_{k} \equiv 1\left(\bmod 2^{m}\right) \Leftrightarrow k \equiv 0\left(\bmod 2^{m-2}\right) .
\end{aligned}
$$

In the case of degree $n \geqq 2$, we can obtain following results.

THEOREM 2.1. Assume that $k>n+1$.

(1) Suppose that $p \neq 2$ is a regular prime. Then we get

$$
\Psi_{k} \equiv 1\left(\bmod p^{m}\right) \Leftrightarrow k \equiv 0\left(\bmod p^{m-1}(p-1)\right)
$$


(2) Let $n=2$ and $Z_{m}$ be the numerator of the $m$-th Bernoulli number $B_{m}$. If $p \neq 2,3$ and $Z_{p-3} \neq \equiv(\bmod p)$, then we get

$$
\Psi_{k} \equiv 1\left(\bmod p^{m}\right) \Leftrightarrow k \equiv 0\left(\bmod p^{m-1}(p-1)\right) .
$$

In both (1) and (2), we should remark that the condition of the left hand side always implies the condition of the right hand side for all odd prime numbers $p$.

Proof. (1) We refer the following result from [9]. Let $\Psi_{k}(Z)$ $=\sum a_{k}(T) \exp \{2 \pi i \operatorname{tr}(T Z)\}$ be the Fourier expansion of $\Psi_{k}$. If $T$ is a non zero matrix and $p \neq 2$, then the rational number

$$
b_{k}(T)=a_{k}(T) \cdot \frac{B_{k}}{k} \cdot \prod_{\nu=1}^{r(T)} \frac{b_{\nu} B_{2 \nu}}{\nu} \cdot \prod_{\mu=r(T)+1}^{k-1} \frac{B_{2 \mu}}{\mu}
$$

is a $p$-adic integer, where $b_{m}$ is the denominator of $\frac{B_{2 m}}{m}$ and $\gamma(T)$ is an integer which depends on $T$ (cf. [9]).

If we put

$$
c_{k}(T)=\prod_{\nu=1}^{r(T)} \frac{b_{\nu} B_{2 \nu}}{\nu} \cdot \prod_{\mu=r(T)+1}^{k-1} \frac{B_{2 \mu}}{\mu}
$$

then we obtain

$$
\Psi_{k}(T)=1+\frac{k}{B_{k}} \sum_{T \neq 0} \frac{b_{k}(T)}{c_{k}(T)} \exp \{2 \pi i \operatorname{tr}(T Z)\}
$$

The proof of $(\Leftarrow)$. Let $\nu_{p}$ be the normalized, $p$-adic additive valuation of $\boldsymbol{Q}_{p}$. First, we estimate the value $\nu_{p}\left(k / B_{k}\right)$. Since $k \equiv 0$ $\left(\bmod p^{m-1}(p-1)\right)$, we can apply the von Staudt's theorem and obtain

$$
\nu_{p}\left(k / B_{k}\right)=\nu_{p}(k)-\nu_{p}\left(B_{k}\right) \geqq(m-1)-(-1)=m .
$$

Next, we shall estimate the value $\nu_{p}\left(c_{k}(T)\right)$. It is well known in number theory that prime number $p$ is regular if and only if $p$ doesn't appear in the numerators of the Bernoulli numbers $B_{2}, B_{4}, \cdots, B_{p-3}$. Using Kummer's congruences for Bernoulli numbers and the above fact, we see that

$$
\nu_{p}\left(\prod_{\nu=1}^{k-1}\left(B_{2 \nu} / \nu\right)\right) \leqq 0
$$


Therefore we obtain $\nu_{p}\left(c_{k}(T)\right) \leqq 0$. Thus we get $\nu_{p}\left(b_{k}(T) / c_{k}(T)\right) \geqq 0$, and $\Psi_{k} \equiv 1\left(\bmod p^{m}\right)$.

The proof of $(\Rightarrow)$. Since we assume $\Psi_{k} \equiv 1\left(\bmod p^{m}\right)$, we see $\Phi^{n-1}\left(\Psi_{k}\right)=E_{k} \equiv 1\left(\bmod p^{m}\right)$. By the result of the case of degree 1 , we obtain $k \equiv 0\left(\bmod p^{m-1}(p-1)\right)$.

It is obvious from the above proof that the left hand side always implies the right hand side without the condition of regularity for prime number $p$.

(2) In the case $n=2$, Maass has proved the following result (cf. [6]).

Let $N_{m}$ be the denominator of the $m$-th Bernoulli number $B_{m}$. We assume that $k \equiv 0(\bmod 2), k>3$ and $T>0$. Then

$$
a_{k}(T) \cdot \frac{B_{k}}{k} \cdot \frac{q \cdot B_{2 k-2}}{2 k-2}
$$

is a rational integer, where $q$ is the greatest divisor of $(k-1) N_{2 k-2}$, whose prime factors $p$ satisfy $p \equiv-1(\bmod 4)$ and $N_{2 k-2} \equiv 0(\bmod p)$. From this, if we write

$$
\begin{aligned}
\Psi_{k}(Z)=1 & +\frac{k(2 k-2)}{q \cdot B_{k} \cdot B_{2 k-2}} \sum_{T>0} b_{k}(T) \exp \{2 \pi i \operatorname{tr}(T Z)\} \\
& +\frac{2 k}{B_{k}} \sum_{\substack{\operatorname{det} T^{\prime}=0 \\
T^{\prime} \neq 0}} b_{k}^{\prime}\left(T^{\prime}\right) \exp \left\{2 \pi i \operatorname{tr}\left(T^{\prime} Z\right)\right\},
\end{aligned}
$$

then $b_{k}(T), b_{k}^{\prime}\left(T^{\prime}\right) \in Z$. Here, we assume $k \equiv 0\left(\bmod p^{m-1}(p-1)\right)$. Then we obtain $\nu_{p}\left(k / B_{k}\right) \geqq m$ as in (1). Using the condition $Z_{p-3} \neq 0(\bmod p)$, we can get following inequality.

$$
\begin{aligned}
\nu_{p}\left(\frac{k(2 k-2)}{q \cdot B_{k} \cdot B_{2 k-2}}\right) & =\nu_{p}\left(\frac{k}{B_{k}}\right)+\nu_{p}\left(\frac{2 k-2}{q \cdot B_{2 k-2}}\right) \\
& \geqq \nu_{p}\left(\frac{k}{B_{k}}\right) \geqq m .
\end{aligned}
$$

This shows that $\Psi_{k} \equiv 1\left(\bmod p^{m}\right)$. Now the rest of the proof of (2) is the same as (1). Thus we completed the proof of Theorem 2.1.

Remark. We have seen that the condition $Z_{p-3} \neq 0(\bmod p)$ is valid for all prime numbers $p$ smaller than 4001 (cf. [1]). Obviously, if $p$ is regular, then $Z_{p-3} \neq 0(\bmod p)$. We will show in Appendix that there exists a prime $p$ which does not satisfy the condition of (2) in Theorem 2.1 and, for this $p, \Psi_{p-1} \not \equiv 1(\bmod p)$. 


\section{Part II}

\section{§1. Fourier expansion of Siegel modular forms of degree 2}

Let $\boldsymbol{Q}\left\{q_{0}, q_{1}, q_{2}\right\}^{+}$denote the ring of all formal power series of the form

$$
\begin{aligned}
& \sum_{T=\left(\begin{array}{cc}
t_{0} & \frac{t_{1}}{2} \\
\frac{t_{1}}{2} & t_{2}
\end{array}\right) \geqq 0} a(T) \exp \{2 \pi i \operatorname{tr}(T Z)\}=\sum a(T) q_{0}^{t_{0}} q_{1}^{t_{1}} q_{2}^{t_{2}} \\
& \left(a(T) \in \boldsymbol{Q}, Z=\left(\begin{array}{ll}
z_{0} & z_{1} \\
z_{1} & z_{2}
\end{array}\right), q_{j}=\exp \left(2 \pi i z_{j}\right)\right)
\end{aligned}
$$

where $T$ runs over all half integral positive semi-definite symmetric matrices.

Let $\mathfrak{D}_{p}\left\{q_{0}, q_{1}, q_{2}\right\}^{+}$be the subring of $\boldsymbol{Q}\left\{q_{0}, q_{1}, q_{2}\right\}^{+}$consisting of all elements of $\boldsymbol{Q}\left\{q_{0}, q_{1}, q_{2}\right\}^{+}$with $a(T) \in \mathfrak{S}_{p}=\boldsymbol{Q} \cap \boldsymbol{Z}_{p}$. For any element $f\left(q_{0}, q_{1}, q_{2}\right)$ $=\sum a(T) q_{0}^{t_{0}} q_{1}^{t_{1}} q_{2}^{t_{2}}$ of $\boldsymbol{Q}\left\{q_{0}, q_{1}, q_{2}\right\}^{+}$, we define $\tilde{f}$ by $\tilde{f}\left(q_{0}, q_{1}, q_{2}\right)=\sum \widetilde{a(T)} q_{0}^{t_{0}} q_{1}^{t_{1}} q_{2}^{t_{2}}$ where the tilde denotes the reduction $\bmod p$, and denote by $\boldsymbol{F}_{p}\left\{q_{0}, q_{1}, q_{2}\right\}^{+}$ the $\boldsymbol{F}_{p}$-algebra consisting of $\tilde{f}$ with $f$ in $\mathfrak{D}_{p}\left\{q_{0}, q_{1}, q_{2}\right\}^{+}$.

In the rest of this paper, we shall mainly deal with the case of degree 2.

First of all, we shall define a linear order among the half integral positive semi-definite symmetric matrices $T=\left[\begin{array}{ll}t_{0} & \frac{t_{1}}{2} \\ \frac{t_{1}}{2} & t_{2}\end{array}\right]$ as follows:

1. We arrange in order of $\operatorname{tr}(T)$.

2. When the traces are equal, we arrange them in order of $t_{0}$.

3. When both the traces and $t_{0}$ 's are equal, we arrange in order of $t_{1}$.

We arrange the half integral positive semi-definite symmetric matrices $T$, and write them $T_{0}, T_{1}, T_{2}, \ldots$ according to this order. Then

$$
f(Z)=\sum_{n=0}^{\infty} a\left(T_{n}\right) \exp \left\{2 \pi i \operatorname{tr}\left(T_{n} Z\right)\right\}
$$

Here, we shall prove some lemma which is required later.

LEMMA 1.1. Let $p$ be a prime number. Suppose $f, g \in \mathcal{D}_{p}\left\{q_{0}, q_{1}, q_{2}\right\}^{+}$ and $h \in \boldsymbol{Q}\left\{q_{0}, q_{1}, q_{2}\right\}^{+}$. Furthermore, we assume that the first non zero 
coefficient of $g$ is a p-adic unit. If $f=g h$, then we get $h \in \mathfrak{D}_{p}\left\{q_{0}, q_{1}, q_{2}\right\}^{+}$.

Proof. Let $g(Z)=\sum_{k=n}^{\infty} a\left(T_{k}\right) \exp \left\{2 \pi i \operatorname{tr}\left(T_{k} Z\right)\right\}\left(a\left(T_{n}\right) \neq 0\right)$ and $h(Z)$ $=\sum_{j=s}^{\infty} b\left(T_{j}\right) \exp \{2 \pi i \operatorname{tr}(T Z)\}\left(b\left(T_{s}\right) \neq 0\right)$ be the series expansions of $f$ and $g$. By our assumption, $a\left(T_{n}\right)$ is a $p$-adic unit. Suppose that $h \notin \mathfrak{O}_{p}\left\{q_{0}, q_{1}, q_{2}\right\}^{+}$. We assume $b\left(T_{m}\right)$ is the first coefficient which does not belong to $\mathfrak{D}_{p}$. Then the coefficient of $\exp \left\{2 \pi i \operatorname{tr}\left(T_{n}+T_{m}\right)\right\}$ in the series expansion of $g(Z) h(Z)$ is $a\left(T_{n}\right) b\left(T_{m}\right)+\sum a\left(T_{j}\right) b\left(T_{k}\right)$, where the sum runs over all matrices $T_{j}$ and $T_{k}(k<m$ and $j>n)$ satisfying $T_{j}+T_{k}$ $=T_{n}+T_{m}$. By our assumption, the second sum of above expression must be contained in $\mathfrak{D}_{p}$. Hence we get $a\left(T_{n}\right) b\left(T_{m}\right) \in \mathfrak{D}_{p}$. Since $a\left(T_{n}\right)$ is a $p$-adic unit, we have $b\left(T_{m}\right) \in \mathfrak{D}_{p}$, which is a contradiction.

\section{§2. The graded ring of modular forms of degree 2}

The structure of the graded ring of modular forms of degree 2 was determined by J. Igusa (cf. [3]). Later, E. Freitag gave an elementary proof of Igusa's result (cf. [2]).

For real vectors $A=\left(\begin{array}{l}a_{1} \\ a_{2}\end{array}\right), B=\left(\begin{array}{l}b_{1} \\ b_{2}\end{array}\right)$, we defined the theta series $\vartheta(Z ; A, B)$ over $\mathrm{H}_{2}$ by

$$
\vartheta(Z ; A, B)=\sum \exp \left[\pi i\left\{{ }^{t}(G+A) Z(G+A)+2^{t} B G\right\}\right]
$$

where the summation is taken over all vectors $G=\left(\begin{array}{l}g_{1} \\ g_{2}\end{array}\right)$ with entries in $\boldsymbol{Z}$.

We define $\vartheta_{i}(Z)(1 \leqq i \leqq 10)$ as follows ;

$$
\begin{aligned}
& \vartheta_{1}(Z)=\vartheta\left(Z ;\left(\begin{array}{l}
0 \\
0
\end{array}\right),\left(\begin{array}{l}
0 \\
0
\end{array}\right)\right), \quad \vartheta_{2}(Z)=\vartheta\left(Z ;\left(\begin{array}{l}
0 \\
0
\end{array}\right),\left(\frac{1}{\frac{1}{2}}\right)\right), \\
& \vartheta_{3}(Z)=\vartheta\left(Z ;\left(\begin{array}{l}
0 \\
0
\end{array}\right),\left(\begin{array}{l}
\frac{1}{2} \\
0
\end{array}\right)\right), \quad \vartheta_{4}(Z)=\vartheta\left(Z ;\left(\begin{array}{l}
0 \\
0
\end{array}\right),\left(\begin{array}{l}
\frac{1}{2} \\
\frac{1}{2}
\end{array}\right)\right), \\
& \vartheta_{5}(Z)=\vartheta\left(Z ;\left(\begin{array}{l}
\frac{1}{2} \\
0
\end{array}\right),\left(\begin{array}{l}
0 \\
0
\end{array}\right)\right), \quad \vartheta_{6}(Z)=\vartheta\left(Z ;\left(\begin{array}{l}
\frac{1}{2} \\
0
\end{array}\right),\left(\begin{array}{l}
0 \\
\frac{1}{2}
\end{array}\right)\right),
\end{aligned}
$$




$$
\begin{aligned}
& \vartheta_{7}(Z)=\vartheta\left(Z ;\left(\begin{array}{l}
0 \\
\frac{1}{2}
\end{array}\right),\left(\begin{array}{l}
0 \\
0
\end{array}\right)\right), \quad \vartheta_{8}(Z)=\vartheta\left(Z ;\left(\begin{array}{c}
0 \\
\frac{1}{2}
\end{array}\right),\left(\begin{array}{c}
\frac{1}{2} \\
0
\end{array}\right)\right), \\
& \vartheta_{9}(Z)=\vartheta\left(Z ;\left(\begin{array}{l}
\frac{1}{2} \\
\frac{1}{2}
\end{array}\right),\left(\begin{array}{l}
0 \\
0
\end{array}\right), \quad \vartheta_{10}(Z)=\vartheta\left(Z ;\left(\begin{array}{l}
\frac{1}{2} \\
\frac{1}{2}
\end{array}\right),\left(\begin{array}{c}
\frac{1}{2} \\
\frac{1}{2}
\end{array}\right)\right) .\right.
\end{aligned}
$$

Now we can state the theorems of Igusa and Freitag.

Theorem 2.1 (J. Igusa [4]). Put $\Theta_{1}(Z)=3^{2} \sum_{i=1}^{10} \vartheta_{i}^{24}(Z)-2^{2} \cdot 11 \Psi_{4}^{3}(Z)$ $+2^{3} \Psi_{6}^{2}(Z)$. Then

(1) $\Theta_{1}(Z)$ is a cusp form of weight 12.

(2) $\chi_{12}(Z)=2^{-15} \cdot 3^{-4} \cdot 11^{-1} \Theta_{1}(Z)$, where $\chi_{12}$ is the cusp form which is defined in Part I, §1.

TheOREM 2.2 (E. Freitag). Put $\Theta_{2}(Z)=\prod_{i=1}^{10} \vartheta_{i}^{2}(Z)$, then we have

(1) $\Theta_{2}(Z)$ is a cusp form of weight 10.

(2) $\chi_{10}(Z)=\Theta_{2}(Z)$, where $\chi_{10}$ is the cusp form which is defined in Part I, §1.

(3) $\Theta_{2}(Z)$ vanishes on $\left\{\left(\begin{array}{ll}z_{0} & z_{1} \\ z_{1} & z_{2}\end{array}\right) \in \boldsymbol{H}_{2} \mid z_{1}=0\right\}$.

(4) If $f(Z)$ is a modular form of even weight $k$ such that $f\left(\begin{array}{cc}z_{0} & 0 \\ 0 & z_{2}\end{array}\right)$ $=0$ (identically), then $f(Z) / \Theta_{2}(Z)$ is a modular form of weight $(k-10)$.

Let $A_{k}$ be the vector space over $C$ of modular forms of even weight $k$. Then the graded ring $A=\oplus_{k \text { : even }} A_{k}$ will be called the graded ring of modular forms of degree 2 and of even weight. Using the result of E. Witt (cf. [11]), E. Freitag gave the following lemma.

LEMMA 2.3. (1) If $f(Z) \in A_{k}$, then we have

$$
f\left(\begin{array}{cc}
z_{0} & 0 \\
0 & z_{2}
\end{array}\right)=\sum_{4 a+6 b+12 c=k} \gamma_{a b c} \Psi_{4}^{a}\left(\begin{array}{cc}
z_{0} & 0 \\
0 & z_{2}
\end{array}\right) \Psi_{6}^{b}\left(\begin{array}{cc}
z_{0} & 0 \\
0 & z_{2}
\end{array}\right) \Psi_{12}^{c}\left(\begin{array}{cc}
z_{0} & 0 \\
0 & z_{2}
\end{array}\right)
$$

with $\gamma_{a b c} \in C$.

(2) If $f(Z) \in A_{k}$, then $f(Z)-P\left(\Psi_{4}(Z), \Psi_{6}(Z), \Psi_{12}(Z)\right)$ vanishes on $\left\{\left(\begin{array}{ll}z_{0} & z_{1} \\ z_{1} & z_{2}\end{array}\right)\right.$ 
$\left.\in \boldsymbol{H}_{2} \mid z_{1}=0\right\}$ for a suitable polynomial $P$.

In relation to the above fact, we shall give some examples which is required later.

$$
\left\{\begin{array}{c}
\Psi_{4}\left(\begin{array}{cc}
z_{0} & 0 \\
0 & z_{2}
\end{array}\right)=E_{4}\left(z_{0}\right) E_{4}\left(z_{2}\right), \quad \Psi_{6}\left(\begin{array}{cc}
z_{0} & 0 \\
0 & z_{2}
\end{array}\right)=E_{6}\left(z_{0}\right) E_{6}\left(z_{2}\right), \\
\Psi_{12}\left(\begin{array}{cc}
z_{0} & 0 \\
0 & z_{2}
\end{array}\right)= \\
\quad c_{1}\left(E_{4}\left(z_{0}\right) E_{4}\left(z_{2}\right)\right)^{3}+c_{2}\left(E_{6}\left(z_{0}\right) E_{6}\left(z_{2}\right)\right)^{2} \\
\quad+c_{3}\left(E_{4}^{3}\left(z_{0}\right) E_{6}^{2}\left(z_{2}\right)+E_{6}^{2}\left(z_{0}\right) E_{4}^{3}\left(z_{2}\right)\right) \cdot \\
c_{1}=\frac{3 \cdot 7^{3} \cdot 29 \cdot 733}{131 \cdot 593 \cdot 691}, \quad c_{2}=\frac{2^{5} \cdot 5^{3} \cdot 1759}{131 \cdot 593 \cdot 691}, \quad c_{3}=\frac{2 \cdot 3 \cdot 5^{3} \cdot 7^{2} \cdot 337}{131 \cdot 593 \cdot 691}
\end{array}\right.
$$

From these relations, we get

$$
\begin{gathered}
\Delta\left(z_{0}\right) \Delta\left(z_{2}\right)=\chi_{12}\left(\begin{array}{cc}
z_{0} & 0 \\
0 & z_{2}
\end{array}\right), \\
E_{4}^{3}\left(z_{0}\right) \Delta\left(z_{2}\right)+E_{4}^{3}\left(z_{2}\right) \Delta\left(z_{0}\right)= \\
e^{-1} \cdot \Psi_{4}^{3}\left(\begin{array}{cc}
z_{0} & 0 \\
0 & z_{2}
\end{array}\right)-e^{-1} \cdot \Psi_{6}^{2}\left(\begin{array}{cc}
z_{0} & 0 \\
0 & z_{2}
\end{array}\right) \\
+e^{-1} \cdot \chi_{12}\left(\begin{array}{cc}
z_{0} & 0 \\
0 & z_{2}
\end{array}\right),
\end{gathered}
$$

where $\Delta(z)=e^{-1} \cdot\left(E_{4}^{3}(z)-E_{6}^{2}(z)\right)=q \prod_{n=1}^{\infty}\left(1-q^{n}\right)^{24}$ is a cusp form of weight $12, e=2^{6} \cdot 3^{3}$ and $q=\exp (2 \pi i z)$.

Making use of Theorem 2.2 (4) and Lemma 2.3, we get the following theorem.

THEOREM 2.4 (J. Igusa). If $f(Z) \in A_{k}$, then $f(Z)$ can be expressed as an isobaric polynomial of $\Psi_{4}(Z), \Psi_{6}(Z), \chi_{10}(Z)$ and $\chi_{12}(Z)$. Namely, $A \cong C\left[\Psi_{4}, \Psi_{6}, \chi_{10}, \chi_{12}\right]$. (As a matter of course, $\Psi_{4}, \Psi_{6}, \chi_{10}$ and $\chi_{12}$ are independent over $\boldsymbol{C}$, mutually (cf. [3])).

\section{§3. $P$-integral modular forms}

Let $\boldsymbol{I}_{k, p}$ be the $\mathfrak{D}_{p}$-module of Siegel modular forms of degree 2 and of even weight $k$ whose Fourier expansions have all their coefficients in $\mathfrak{D}_{p}=\boldsymbol{Q} \cap Z_{p}$.

LEMMA 3.1. (1) We have $\Psi_{4} \in I_{4, p}, \Psi_{6} \in I_{6, p}$ and $\chi_{10} \in I_{10, p}$ for all prime numbers $p$. 
(2) If $p \neq 2,3$, then we have $\chi_{12} \in I_{12, p}$.

Proof. (1) Let $N_{m}$ be the denominator of the $m$-th Bernoulli number $B_{m}$ as in Part I, $\S 2$. From the proof of Part I, Theorem 2.1, we see that

$$
\begin{aligned}
\Psi_{k}(Z)=1 & +\frac{k(2 k-2)}{q \cdot B_{k} \cdot B_{2 k-2}} \sum_{T>0} b_{k}(T) \exp \{2 \pi i \operatorname{tr}(T Z)\} \\
& +\frac{2 k}{B_{k}} \sum_{\substack{\operatorname{det} T^{\prime}=0 \\
T^{\prime} \neq 0}} b_{k}^{\prime}\left(T^{\prime}\right) \exp \left\{2 \pi i \operatorname{tr}\left(T^{\prime} Z\right)\right\}
\end{aligned}
$$

where $q$ is the factor of $(k-1) N_{2 k-2}$ and $b_{k}(T)$ and $b_{k}^{\prime}\left(T^{\prime}\right)$ are rational integers. Since $B_{4}=-1 / 30, B_{6}=1 / 42$ and $B_{10}=5 / 66$, we have $\Psi_{4} \in I_{4, p}$ and $\Psi_{6} \in \boldsymbol{I}_{6, p}$ for all prime numbers $p$. From the result of Part II, Theorem 2.2 and the definition of the theta series $\vartheta_{i}(Z)$, we see that all the Fourier coefficients of $\chi_{10}$ are algebraic integers. Moreover, it follows from the definition of $\chi_{10}$ that all the Fourier coefficients of $\chi_{10}$ are rational numbers. Therefore, we see that all the Fourier coefficients of $\chi_{10}$ are rational integers. This shows that $\chi_{10} \in I_{10, p}$ for all prime numbers $p$.

(2) It follows from Part II, Theorem 2.1 that all the Fourier coefficients of $\Theta_{1}(Z)$ are rational integers. Namely, $\chi_{12}$ has the $p$-integral Fourier coefficients if $p \neq 2,3,11$. However, we can see from the definition of $\chi_{12}$ that all the Fourier coefficients of $\chi_{12}$ are $p$-integral if $p$ $\neq 2,3,5,7$ and 337. Therefore, if $p \neq 2,3$, then all the Fourier coefficients of $\chi_{12}$ are $p$-integral. This completes the proof.

Proposition 3.2. Let $p \neq 2,3$ be a prime number.

(1) If $f(Z) \in \boldsymbol{I}_{k, p}$, then we have

$$
f\left(\begin{array}{cc}
z_{0} & 0 \\
0 & z_{2}
\end{array}\right)=\sum_{4 a+6 b+12 c=k} \gamma_{a b c} \Psi_{4}^{a}\left(\begin{array}{cc}
z_{0} & 0 \\
0 & z_{2}
\end{array}\right) \Psi_{6}^{b}\left(\begin{array}{cc}
z_{0} & 0 \\
0 & z_{2}
\end{array}\right) \chi_{12}^{c}\left(\begin{array}{cc}
z_{0} & 0 \\
0 & z_{2}
\end{array}\right)
$$

with $\gamma_{a b c} \in \mathfrak{D}_{p}$.

(2) If $f(Z) \in I_{k, p}$, then we have

$$
f(Z)=\sum_{4 a+6 b+10 c+12 d=k} \omega_{a b c d} \Psi_{4}^{a}(Z) \Psi_{6}^{b}(Z) \chi_{10}^{c}(Z) \chi_{12}^{d}(Z)
$$

with $\omega_{a b c d} \in \mathfrak{D}_{p}$. 
Proof. (1) By Lemma 2.3 and (2.1), we have a following expression,

$$
\begin{aligned}
f\left(\begin{array}{cc}
z_{0} & 0 \\
0 & z_{2}
\end{array}\right)= & \sum \rho_{a b c}\left(E_{4}\left(z_{0}\right) E_{4}\left(z_{2}\right)\right)^{a} \cdot\left(E_{6}\left(z_{0}\right) E_{6}\left(z_{2}\right)\right)^{b} \\
& \times\left(E_{4}^{3}\left(z_{0}\right) E_{6}^{2}\left(z_{2}\right)+E_{6}^{2}\left(z_{0}\right) E_{4}^{3}\left(z_{2}\right)\right)^{c}
\end{aligned}
$$

with $\rho_{a b c} \in C$.

Now, put $4 a+6 b+12 c=k=2 k^{\prime}$, then $k^{\prime} \equiv b(\bmod 2)$. First assume that $k^{\prime}$ is even. Substituting $E_{6}^{2}(z)$ by $E_{4}^{3}(z)-e \cdot \Delta(z)$ with $e=2^{6} \cdot 3^{3}$ in the above expression, we have

$$
f\left(\begin{array}{cc}
z_{0} & 0 \\
0 & z_{2}
\end{array}\right)=\sum_{4 a+12 b=4 c+12 d=k} \delta_{a b c d} E_{4}^{a}\left(z_{0}\right) E_{4}^{c}\left(z_{2}\right) \Delta^{b}\left(z_{0}\right) \Delta^{d}\left(z_{2}\right), \quad \delta_{a b c d} \in C
$$

By comparing the Fourier coefficients of both sides, we get $\delta_{a b c d} \in \mathscr{D}_{p}$ if $p \neq 2,3$.

Since $f\left(\begin{array}{cc}z_{0} & 0 \\ 0 & z_{2}\end{array}\right)=f\left(\begin{array}{cc}z_{2} & 0 \\ 0 & z_{0}\end{array}\right), f\left(\begin{array}{cc}z_{0} & 0 \\ 0 & z_{2}\end{array}\right)$ can be expressed as $\mathfrak{D}_{p}$-linear combination of the terms

$$
E_{4}^{a}\left(z_{0}\right) E_{4}^{c}\left(z_{2}\right) \Delta^{b}\left(z_{0}\right) \Delta^{d}\left(z_{2}\right)+E_{4}^{c}\left(z_{0}\right) E_{4}^{a}\left(z_{2}\right) \Delta^{d}\left(z_{0}\right) \Delta^{b}\left(z_{2}\right)
$$

with $4 a+12 b=4 c+12 d=k$. Furthermore, as the terms with the suitable power of $E_{4}\left(z_{0}\right) E_{4}\left(z_{2}\right)$ and $\Delta\left(z_{0}\right) \Delta\left(z_{2}\right)$ are combined together, we can verify that $f\left(\begin{array}{cc}z_{0} & 0 \\ 0 & z_{2}\end{array}\right)$ is expressed as an isobaric polynomial of

$$
E_{4}\left(z_{0}\right) E_{4}\left(z_{2}\right), \quad \Delta\left(z_{0}\right) \Delta\left(z_{2}\right), \quad E_{4}^{a}\left(z_{0}\right) \Delta^{b}\left(z_{2}\right)+E_{4}^{a}\left(z_{2}\right) \Delta^{b}\left(z_{0}\right) \quad(4 a=12 b)
$$

with coefficients in $\mathfrak{D}_{p}$.

The last term is nothing but $\left(E_{4}^{3}\left(z_{0}\right) \Delta\left(z_{2}\right)\right)^{m}+\left(E_{4}^{3}\left(z_{2}\right) \Delta\left(z_{0}\right)\right)^{m}$, hence $f\left(\begin{array}{cc}z_{0} & 0 \\ 0 & z_{2}\end{array}\right)$ can be expressed as an isobaric polynomial of

$$
E_{4}\left(z_{0}\right) E_{4}\left(z_{2}\right), \quad \Delta\left(z_{0}\right) \Delta\left(z_{2}\right), \quad E_{4}^{3}\left(z_{0}\right) \Delta\left(z_{2}\right)+E_{4}^{3}\left(z_{2}\right) \Delta\left(z_{0}\right)
$$

with coefficients in $\mathfrak{D}_{p}$.

By (2.2) and (2.3) in $\S 2$, we conclude that $f\left(\begin{array}{cc}z_{0} & 0 \\ 0 & z_{2}\end{array}\right)$ can be expressed as an isobaric polynomial of $\Psi_{4}\left(\begin{array}{cc}z_{0} & 0 \\ 0 & z_{2}\end{array}\right), \Psi_{6}\left(\begin{array}{cc}z_{0} & 0 \\ 0 & z_{2}\end{array}\right)$ and $\chi_{12}\left(\begin{array}{cc}z_{0} & 0 \\ 0 & z_{2}\end{array}\right)$ with coefficients in $\mathfrak{D}_{p}$ if $p \neq 2,3$. 
If $k^{\prime}$ is odd, $b$ is also odd. By multiplying $E_{6}^{-1}\left(z_{0}\right) E_{6}^{-1}\left(z_{2}\right)$ to both sides of (3.1), we see

$$
f\left(\begin{array}{cc}
z_{0} & 0 \\
0 & z_{2}
\end{array}\right) \cdot E_{6}^{-1}\left(z_{0}\right) E_{6}^{-1}\left(z_{2}\right)=\sum \rho_{a b c} \Psi_{4}^{a}\left(\begin{array}{cc}
z_{0} & 0 \\
0 & z_{2}
\end{array}\right) \Psi_{6}^{b-1}\left(\begin{array}{cc}
z_{0} & 0 \\
0 & z_{2}
\end{array}\right) \chi_{12}^{c}\left(\begin{array}{cc}
z_{0} & 0 \\
0 & z_{2}
\end{array}\right)
$$

with $\rho_{a b c} \in C$. Now, the Fourier coefficients of the left hand side belong to $\mathfrak{D}_{p}$. Therefore the same argument is applicable to this case.

(2) Let $f \in I_{k, p}$. Then by (1), we see that $f(Z)-P\left(\Psi_{4}(Z), \Psi_{6}(Z), \chi_{12}(Z)\right.$ ) vanishes on $\left\{\left(\begin{array}{ll}z_{0} & z_{1} \\ z_{1} & z_{2}\end{array}\right) \in \boldsymbol{H}_{2} \mid z_{1}=0\right\}$ for suitable $\mathfrak{D}_{p}$-polynomial $P$. Therefore $f(Z)-P\left(\Psi_{4}(Z), \Psi_{6}(Z), \chi_{12}(Z)\right)=\chi_{10}(Z) h(Z)$ for some $h \in A_{k-10} \cap Q\left\{q_{0}, q_{1}, q_{2}\right\}^{+}$. It follows from Part II, Lemma 1.1 that $h(Z)$ is an element of $\boldsymbol{I}_{k-10, p}$. By induction, we can see that $f(Z)$ is expressed as an isobaric polynomial of $\Psi_{4}(Z), \Psi_{6}(Z), \chi_{10}(Z)$ and $\chi_{12}(Z)$ with coefficients in $\mathfrak{D}_{p}$. Thus we have proved our theorem.

\section{§4. The structure of the algebra of $\operatorname{modular}$ forms $\bmod p$}

Let $\tilde{I}_{k, p}$ be the $\boldsymbol{F}_{p}$-vector space of all formal power series $\sum \widetilde{a\left(T^{\prime}\right)}$ $\exp \{2 \pi i \operatorname{tr}(T Z)\}=\sum \widetilde{a(T)} q_{0}^{t_{0}} q_{1}^{t_{1}} q_{2}^{t_{2}}$ obtained from elements $f(Z)=\sum a(T)$ $\exp \{2 \pi i \operatorname{tr}(T Z)\}$ of $\boldsymbol{I}_{k, p}$ by reducing the coefficients $\bmod p$.

We define the $\boldsymbol{F}_{p}$-subalgebra $\tilde{\boldsymbol{M}}_{2}$ of $\boldsymbol{F}_{p}\left\{q_{0}, q_{1}, q_{2}\right\}^{+}$by $\boldsymbol{M}_{2}=\sum_{k \text { :even }} \tilde{\boldsymbol{I}}_{k, p}$, which is called the algebra of Siegel modular forms mod $p$ of degree 2.

We can similarly define the $\boldsymbol{F}_{p}$-algebra $\tilde{M}_{1}$ of elliptic modular forms $\bmod p$ as in [10]. The structure of $\tilde{M}_{1}$ is determined by H. P. F. Swinnerton-Dyer as follows.

THEOREM 4.1 (Swinnerton-Dyer [10]). (1) Suppose that $p \geqq 5$. Then $\tilde{M}_{1} \cong \boldsymbol{F}_{p}[Q, R] /(\tilde{A}-1)$ where $A(Q, R)$ is a $\mathfrak{D}_{p}$-polynomial defined by $E_{p-1}$ $=A\left(E_{4}, E_{6}\right)$.

(2) Suppose that $p=2$ or 3 . Then $\tilde{M}_{1}=F_{p}[\tilde{\Delta}]$.

The main purpose of this section is to determine the structure of $\tilde{M}_{2}$.

Until the end of the proof of Lemma 4.3, we assume $p \geqq 5$. It follows from the results of $\S 3$ that there is a ring homomorphism

$$
\mathfrak{S}_{p}[U, V, W, X] \longrightarrow \boldsymbol{F}_{p}[U, V, W, X] \stackrel{\pi^{\prime}}{\longrightarrow} \tilde{M}_{2}
$$


where the left hand arrow is the extension of $\mathfrak{D}_{p} \rightarrow F_{p}$ and $\pi^{\prime}$ is defined by corresponding $U, V, W$ and $X$ to $\tilde{\Psi}_{4}, \tilde{\Psi}_{6}, \tilde{\chi}_{10}$ and $\tilde{\chi}_{12}$. Since $\pi^{\prime}$ is surjective, to determine the structure of $\tilde{M}_{2}$ we have only to determine the kernel of $\pi^{\prime}$.

The following diagram is commutative.

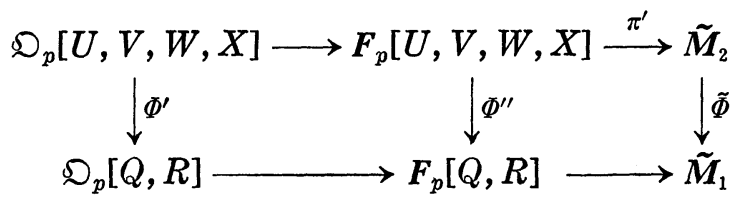

where $\Phi^{\prime}$ and $\Phi^{\prime \prime}$ are the ring homomorphisms defined by $U \mapsto Q, V \mapsto R, W$ $\mapsto 0$ and $X \mapsto 0$, and $\tilde{\Phi}$ is the ring homomorphism defined by $\tilde{\Phi}\left(\tilde{f}\left(q_{0}, q_{1}, q_{2}\right)\right)$ $=\tilde{f}\left(q_{0}, 1,0\right)$ for any $\tilde{f}\left(q_{0}, q_{1}, q_{2}\right) \in \tilde{M}_{2}$. It is easy to show that $\tilde{\Phi}$ is surjective.

LEMMA 4.2. Krull dim. $\tilde{M}_{2}=3$.

Proof. Since ker $\pi^{\prime}$ is non trivial, it is enough to show that Krull $\operatorname{dim} . \tilde{M}_{2} \geqq 3$. Since $\tilde{\Phi}$ is surjective, we obtain $\tilde{M}_{2} / \operatorname{ker} \tilde{\Phi} \cong \tilde{M}_{1}$. From Theorem 4.1, we have Krull $\operatorname{dim} . \tilde{M}_{1}=1$. Hence there exists a following sequence of prime ideals;

$$
0 \subseteq \operatorname{ker} \tilde{\Phi} \subseteq \mathfrak{p} \subseteq \tilde{M}_{2} .
$$

We consider the following ideal of $\tilde{M}_{2}$;

$$
\mathfrak{p}^{\prime}=\left\{\tilde{f}\left(q_{0}, q_{1}, q_{2}\right) \in \tilde{M}_{2} \mid \hat{f}\left(q_{0}, 1, q_{2}\right)=0\right\} .
$$

Using the fact that the ring of formal power series $F_{p}[[X, Y]]$ is an integral domain, we obtain that $\mathfrak{p}^{\prime}$ is prime. Since $0 \neq \tilde{\chi}_{10} \in \mathfrak{p}^{\prime}, \mathfrak{p}^{\prime}$ is a non zero ideal. It follows from $\operatorname{ker} \tilde{\Phi}=\left\{\tilde{f}\left(q_{0}, q_{1}, q_{2}\right) \in \tilde{M}_{2} \mid \tilde{f}\left(q_{0}, 1,0\right)=0\right\}$ that $\mathfrak{p}^{\prime} \subset \operatorname{ker} \tilde{\Phi}$. Moreover, since $\tilde{\chi}_{12} \in \operatorname{ker} \tilde{\Phi}$ and $\tilde{\chi}_{12} \notin \mathfrak{p}^{\prime}$, then we get the following sequence of prime ideals;

$$
0 \subseteq \mathfrak{p}^{\prime} \subseteq \operatorname{ker} \tilde{\Phi} \subseteq \mathfrak{p} \subseteq \tilde{M}_{2} .
$$

Then Krull $\operatorname{dim} . \tilde{M}_{2} \geqq 3$. This completes the proof.

From the above lemma, we can see that $\operatorname{ker} \pi^{\prime}$ is a prime ideal of height 1 . We shall determine the structure of this ideal. 
LEMMA 4.3. Let $B$ be the polynomial with coefficients in $\mathfrak{S}_{p}$ satisfying $\Psi_{p-1}=B\left(\Psi_{4}, \Psi_{6}, \chi_{10}, \chi_{12}\right)$ and let $\tilde{B}$ be the polynomial in $\boldsymbol{F}_{p}[U, V, W, X]$ obtained by $B$ reduction $\bmod p$ of coefficients. Then $\tilde{B}-1$ is irreducible in $\boldsymbol{F}_{p}[U, V, W, X]$.

Proof. We assume that $\tilde{B}-1$ is reducible. Then we can write

$$
\tilde{B}-1=\left(\phi_{n}+\phi_{n-1}+\cdots+\phi_{0}\right)\left(\psi_{m}+\psi_{m-1}+\cdots+\psi_{0}\right)
$$

where $\phi_{i}$ and $\psi_{j}$ are isobaric polynomials of weight $i$ and $j$, respectively. From the definition of $\Phi^{\prime \prime}$, we have $\Phi^{\prime \prime}(\tilde{B}-1)=\tilde{A}-1$ where $A$ is polynomial satisfying $E_{p-1}=A\left(E_{4}, E_{6}\right)$. Since (the weight of $\Phi^{\prime \prime}\left(\phi_{n}+\cdots\right)$ ) $+\left(\right.$ the weight of $\left.\Phi^{\prime \prime}\left(\psi_{m}+\cdots\right)\right)=p-1, \Phi^{\prime \prime}\left(\phi_{n}+\cdots\right)$ and $\Phi^{\prime \prime}\left(\psi_{m}+\cdots\right)$ are not constants. This contradicts the fact that $\tilde{A}-1$ is irreducible.

Now we shall fix a prime number $p \neq 2$, 3 satisfying $\Psi_{p-1} \equiv 1(\bmod p)$. Then $\tilde{B}-1$ is contained in ker $\pi^{\prime}$. From the above lemma, $(\tilde{B}-1)$ is a prime ideal. It follows from Lemma 4.2 that $\operatorname{ker} \pi^{\prime}=(\tilde{B}-1)$. Consequently, we obtain the following result.

THEOREM 4.4. Let $p \neq 2,3$ be a prime number satisfying $\Psi_{p-1} \equiv 1$ $(\bmod p)$. Then we obtain

$$
\tilde{M}_{2} \cong \boldsymbol{F}_{p}[U, V, W, X] /(\tilde{B}-1) .
$$

\section{§5. Congruence relations between Siegel modular forms of degree 2}

In this section, we shall study some congruence relations between Siegel modular forms of degree 2.

From now until the end of the proof of Proposition 5.2, we shall fix a prime number $p \neq 2,3$ satisfying $\Psi_{p-1} \equiv 1(\bmod p)$.

Proposition 5.1. Let $f \in \boldsymbol{I}_{k, p}$ and $f^{\prime} \in \boldsymbol{I}_{k^{\prime}, p}$. If we assume that $f \equiv f^{\prime} \neq 0(\bmod p)$, then we have $k \equiv k^{\prime}(\bmod p-1)$.

Proof. Let $f=D\left(\Psi_{4}, \Psi_{6}, \chi_{10}, \chi_{12}\right)$ and $f^{\prime}=D^{\prime}\left(\Psi_{4}, \Psi_{6}, \chi_{10}, \chi_{12}\right)$ where $D$ and $D^{\prime}$ are isobaric polynomials with coefficients in $\mathfrak{D}_{p}$. Furthermore, $\tilde{D}$ and $\tilde{D}^{\prime}$ denote the polynomials obtained from $D$ and $D^{\prime}$ by reduction $\bmod p$. By Theorem 4.4, we obtain $\tilde{D}-\tilde{D}^{\prime} \in(\tilde{B}-1)$, namely $\tilde{D}-\tilde{D}^{\prime}$ $=(\tilde{B}-1)\left(\phi_{m}+\phi_{m-1}+\cdots+\phi_{j}\right)$ where $\phi_{\nu}$ is a isobaric polynomial of weight $\nu$ and $\phi_{m} \neq 0, \phi_{j} \neq 0$. We may assume $k>k^{\prime}$. Comparing the term of same weight of both sides, $\phi_{m-i} \tilde{B}=0$ for $i \neq 0(\bmod p-1)$. 
Since $\phi_{j} \neq 0, m-j \equiv m-k^{\prime} \equiv 0(\bmod p-1)$. Comparing the highest term, we also see that $m+(p-1)=k$. Hence we have $k \equiv k^{\prime}$ $(\bmod p-1)$.

This proposition is a partial generalization in the case of Siegel modular forms of degree 2 of Serre's result [7].

Since $\Psi_{p-1} \equiv 1(\bmod p)$, we have following sequences for any even integer $\alpha(0 \leqq \alpha \leqq p-1)$.

$$
\tilde{\boldsymbol{I}}_{\alpha, p} \subset \tilde{\boldsymbol{I}}_{\alpha+p-1, p} \subset \cdots \subset \tilde{\boldsymbol{I}}_{\alpha+m(p-1), p} \subset \cdots .
$$

If we put $\tilde{I}_{p}^{\alpha}=\bigcup_{m \geqq 0} \tilde{I}_{\alpha+m(p-1), p}$, then we obtain the following.

Proposition 5.2. In the above definition, we obtain $\tilde{M}_{2}=\oplus_{0 \leqq \alpha \leqq p-1} \tilde{\boldsymbol{I}}_{p}^{\alpha}$, namely $\tilde{M}_{2}$ is the graded algebra graded by $Z /(p-1) Z$.

Proof. Let $\tilde{f} \in \tilde{I}_{p}^{\alpha} \cap \tilde{\boldsymbol{I}}_{p}^{\beta}$ and $\tilde{f} \neq 0$. Then $\tilde{f} \in \tilde{\boldsymbol{I}}_{\alpha+m(p-1), p} \cap \tilde{\boldsymbol{I}}_{\beta+m(p-1), p}$ for some integer $m \geqq 0$. Hence we can denote $\tilde{f}=\tilde{g}=\tilde{h} \neq 0$ for $g \in \boldsymbol{I}_{\alpha+m(p-1), p}$ and $h \in \boldsymbol{I}_{\beta+m(p-1), p}$. It follows from previous proposition that $\alpha+m(p-1)$ $\equiv \beta+m(p-1)(\bmod p-1)$. Then $\alpha \equiv \beta(\bmod p-1)$. Consequently, we obtain $\tilde{I}_{p}^{\alpha}=\tilde{I}_{p}^{\beta}$. This completes the proof.

Remark 1. A $p$-adic Siegel modular form can be defined by follows :

For a formal power series $f(Z)=\sum b(T) \exp \{2 \pi i \operatorname{tr}(T Z)\}\left(b(T) \in \boldsymbol{Q}_{p}\right)$, we put $\nu_{p}(f)=\inf _{T \geqq 0} \nu_{p}(b(T))$. Formal power series $g(Z)=\sum a(T)$ $\exp \{2 \pi i \operatorname{tr}(T Z)\}\left(a(T) \in \boldsymbol{Q}_{p}\right)$ is called a $p$-adic Siegel modular form of degree $n$ when there exists a sequence $\left\{f_{i}(Z)\right\}$ of Siegel modular form of degree $n$ with rational Fourier coefficients which satisfy $\nu_{p}\left(g-f_{i}\right)$ $\rightarrow \infty$. Then author studied the property of $p$-adic Siegel modular form, but could not obtain complete results.

Remark 2. The same argument holds in the cases of symmetric Hilbert modular form of real quadratic fields with discriminant 5 and 8.

\section{Appendix}

Recently, the author got the following result in relation to the fact of Part I, §2.

There exists a prime number $p$ satisfying $\Psi_{p-1} \neq 1(\bmod p)$. Indeed, he made sure in case of $p=16843$ that 


$$
a_{p-1}\left(\begin{array}{cc}
1 & \frac{1}{2} \\
\frac{1}{2} & 1
\end{array}\right) \neq 0(\bmod p) .
$$

This fact is obtained by the following argument. Let $\nu_{p}$ be the normalized $p$-adic additive valuation. From the result of Maass [6], we see

$$
a_{k}\left(\begin{array}{cc}
1 & \frac{1}{2} \\
\frac{1}{2} & 1
\end{array}\right)=-\frac{4 k \cdot B_{k-1},\left(\frac{-3}{*}\right)}{B_{k} \cdot B_{2 k-2}}
$$

where $B_{n, x}$ is the generalized Bernoulli number with Dirichlet character $\chi$.

On the other hand, it is known that $p=16843$ satisfies $Z_{p-3} \equiv 0$ $(\bmod p)$ (cf. [5]). We put $k=p-1, p=16843$ in (1). Then we obtain

$$
\nu_{p}\left(-\frac{4(p-1)}{B_{p-1} \cdot B_{2(p-1)-2}}\right) \leqq 0
$$

Next, we shall estimate the value $\nu_{p}\left(B_{(p-1)-1,\left(\frac{-3}{*}\right)}\right)$. In general, the following formula for the generalized Bernoulli number $B_{n, x}$ with Kronecker's symbol $\chi$ holds : Let $f$ be the conductor of $\chi$. If we assume $0<f \leqq p-1$ and $(f, p)=1$, then we have

$$
B_{n, \chi} \equiv \frac{1}{f p} \sum_{a=1}^{f p} \chi(a) a^{n} \quad(\bmod p) .
$$

Therefore, we have

$$
B_{p-2,\left(\frac{-3}{*}\right)} \equiv \frac{1}{3 p} \sum_{b=1}^{3 p}\left(\frac{-3}{b}\right) b^{p-2} \quad(\bmod p) .
$$

But, we have made sure that

$$
\nu_{p}\left(\frac{1}{3 p} \sum_{b=1}^{3 p}\left(\frac{-3}{b}\right) b^{p-2}\right)=0 .
$$

Therefore, we see that $\nu_{p}\left(B_{p-2,\left(\frac{-3}{*}\right)}\right)=0$. Thus we get 


$$
\nu_{p}\left(a_{p-1}\left(\begin{array}{cc}
1 & \frac{1}{2} \\
\frac{1}{2} & 1
\end{array}\right)\right) \leqq 0 .
$$

Consequently, we have $a_{p-1}\left(\begin{array}{cc}1 & \frac{1}{2} \\ \frac{1}{2} & 1\end{array}\right) \neq 0(\bmod p)$ for $p=16843$.

\section{REFERENCES}

[1] Z. I. Borevich and I. R. Shafarevich, Number Theory, Academic Press.

[2] E. Freitag, Zur Theorie der Modulformen zweiten Grades, Nach. Acad. Wiss. Göttingen II, 1965.

[ 3 ] J. Igusa, On Siegel modular forms of genus two (I), Amer. J. Math., 84, 1962.

[4] - On Siegel modular forms of genus two (II), Amer. J. Math., 86, 1964.

[5] W. Johnson, Irregular primes and cyclotomic Invariants, Math. of Computation, 29, 129, 1975.

[ 6 ] H. Maass, Die Fourierkoeffizienten der Eisensteinreihen zweiten Grades, Mat-Fys. Medd. Danske Vid. Selsk., 34, 1964.

[ 7 ] J.-P. Serre, Congruences et formes modulaires, Sem. Bourbaki., 416, 1971/1972.

[ 8 ] — Formes modulaires et fonctions zêta $p$-adiques, Lecture Note in Math., 350, Springer Verlag, 1972.

[ 9 ] C. L. Siegel, Über die Fourierschen Koeffizienten der Eisensteinschen Reihen, MatFys. Medd. Danske Vid. Selsk., 34, 1964.

[10] H. P. F. Swinnerton-Dyer, On $l$-adic representations and congruences for coefficients of modular forms, Lecture Note in Math., 350, Springer Verlag, 1972.

[11] E. Witt, Ein Identität zwischen Modulformen zweiten Grades, Abh. Math. Sem. Hansische Universität., 14, 1941.

Department of Mathematics

Hokkaido University 